\title{
Réalisation d'un montage de projection pour l'ablation submicronique par laser à excimère
}

\author{
F. Weisbuch et S. Lazare
}

Laboratoire de Physicochimie Moléculaire, UMR 5803 du CNRS, Université de Bordeaux I, 351 cours de la Libération, 33405 Talence, France

\begin{abstract}
Résumé: Un montage de projection par laser à excimère $\mathrm{KrF}$ permet la mise en forme de faisceaux microscopiques et leur utilisation pour le micro-usinage par photoablation des surfaces de matériaux, en particulier des polymères. La lentille de projection, de grande ouverture numérique, conduit à une résolution submicronique.
\end{abstract}

\section{INTRODUCTION}

La photoablation par laser à excimère ${ }^{1}$ permet un arrachement de matière très contrôlé à la surface des matériaux, et de ce fait présente un fort potentiel d'applications dans des domaines multidisciplinaires. Ce phénomène s'accommode d'une résolution d'ordre submicronique, à cause du caractère non-thermique des réactions chimiques mises en jeu lors l'interaction lasermatière. La mise en forme du faisceau par projection de masque est une approche bien connue dans le domaine de la microlithographie, qui s'est développé rapidement dans l'industrie de la microélectronique. Contrairement au cas de l'insolation de la résine photosensible, qui nécessite peu d'énergie par impulsion $\left(1-5 \mathrm{~mJ} / \mathrm{cm}^{2}\right)$, le mode photoablation nécessite une intensité au-dessus du seuil d'ablation $\left(>10 \mathrm{~mJ} / \mathrm{cm}^{2}\right)$. Le montage optique et la modélisation des microfaisceaux sont présentés avec quelques exemples d'applications.

\section{MONTAGE OPTIQUE (figure 1)}

La mise en forme du faisceau du laser $\mathrm{KrF}$ (Lambda Physik LPX220i), comprend un système d'éclairement du masque ( $\mathrm{H}=$ homogénéiseur), un masque métallique d'amplitude $(\mathrm{M})$, une lentille de projection $(\mathrm{L})$ du masque et l'échantillon (E). Les optiques du montage ont été réalisées par Francis Goodall ${ }^{2}$, avec des éléments tout en silice et des revêtements antiréfléchissants. La lentille de projection a une ouverture numérique de 0,3 qui permet une résolution d'ordre submicronique, avec un rapport de grandissement de $\times 1 / 10$. L'échantillon est positionné en $\mathrm{x}$ et $\mathrm{y}$ par une table programmable à précision submicronique et en $\mathrm{z}$ manuellement avec une précision du micron. Le champ de mise au point toléré par la lentille de projection, situé à $25 \mathrm{~mm}$ (distance frontale) est de $4 \times 4 \mathrm{~mm}$.

\section{MODELISATION}

La modélisation du profil d'éclairement au niveau de l'échantillon a été obtenu à l'aide du programme Solid-C de Sigma GmbH Munich. Un exemple est donné à la figure 1 pour un faisceau de taille submicronique et donc fortement limité par la diffraction. Le profil d'ablation théorique dans du polycarbonate est obtenu au moyen des courbes d'ablation, connues par ailleurs ${ }^{3}$. La théorie de la modélisation du faisceau (imagerie par projection) peut se trouver dans les références 4 et 5 .

\section{RESULTATS ET CONCLUSION}

Des exemples de gravure par photoablation sont donnés à la figure 2. Des "usinages" par photoablation de haute résolution (submicronique) sont possibles par la technique de projection, puisque la lentille permet la transmission des hautes fréquences spatiales de la modulation de l'intensité. L'arrachement de matière peut être localisé latéralement avec une grande précision, et la profondeur réglée à quelques dizaines de nanomètres près, par la densité d'énergie et la dose (nombre d'impulsions). 

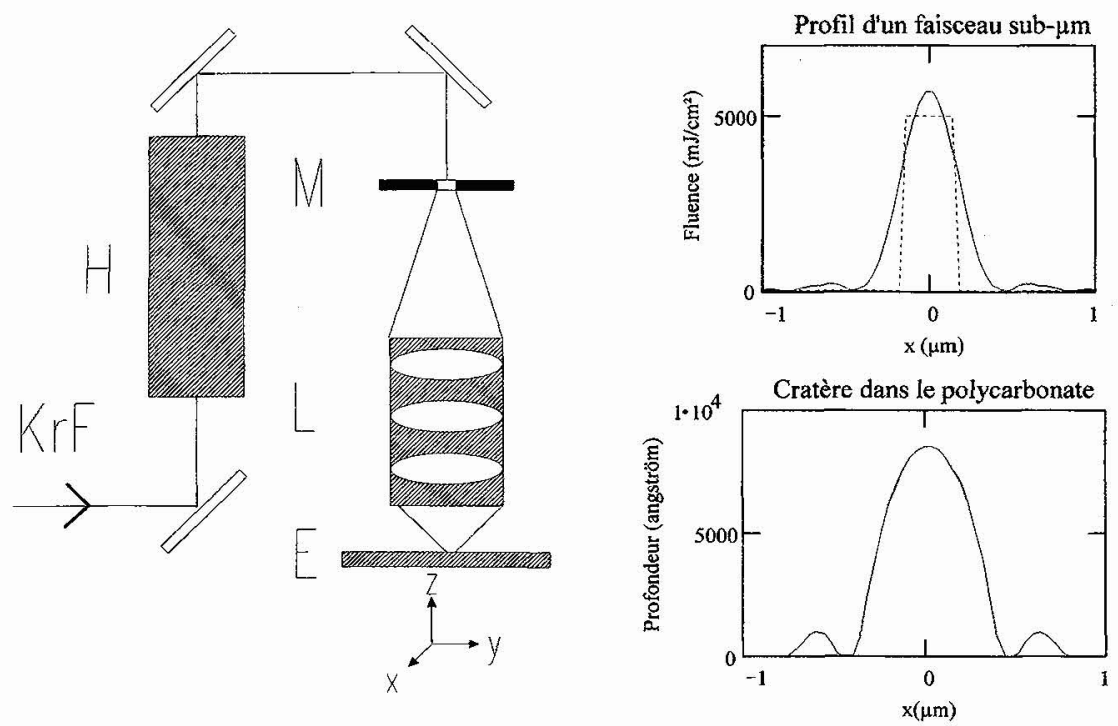

Fig.1 a) Schéma du montage optique b) profil théorique d'un faisceau submicronique calculé pour l'optique utilisée (trait plein), $\sigma=0,2$, par rapport au profil idéal sans diffraction ni aberrations (trait pointillé, $0,35 \mu \mathrm{m}$ de largeur $=$ fenêtre du masque multiplié par le grandissement). Dessous: profil d'ablation correspondant dans le polycarbonate.
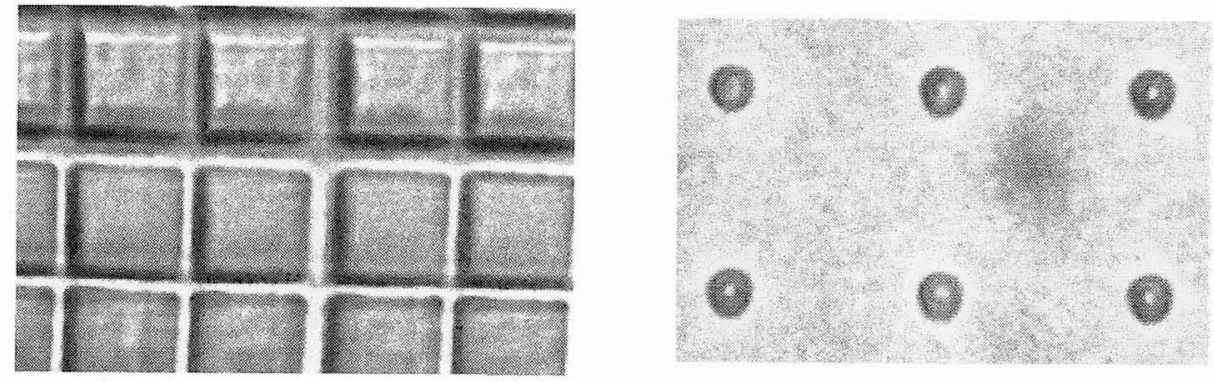

Fig.2 a) Réseau double au pas de $10 \mu \mathrm{m}$ à la surface du polycarbonate. Les lignes les plus étroites sont de dimension légèrement supérieure à $1 \mu \mathrm{m}$ et en relief (les carrés sont en creux): 10 impulsions b) Matrice de trous à la surface du polycarbonate $\Phi=1,5 \mu \mathrm{m}, 500$ impulsions, $\mathrm{p}=3 \mu \mathrm{m}$. Clichés microscopie optique à réflexion.

Remerciements: CNRS pour une AIP SC/SPI et la Région Aquitaine pour le financement. D. Henry et $P$. Schiavonne pour la modélisation.

\section{Références}

1) S. Lazare, D.Drilhole, J.Lopez, F.Weisbuch, Le Vide: science, technique et applications, $\mathbf{n}^{\circ}$ 287 sept.oct.nov. (1998)

2) F.G.Optics, 144 Kings ave., Clapham Park, SW12 OBA. Voir aussi F.N.Goodall, R.A.Lawes, G.C.Arthur, Microelectron.Eng. 11 (1990) 187

3) S.Lazare, V.Granier, Amer.Chem.Soc.Sympos.Ser.Polym.in Microlithography 412 (1989) 411

4) L.F.Thompson, M.J.Bowden ACS Sympos.Series 219 (1983) 15

5) C.A.Mack Microlithography World (1993) Jan.Feb.Mar. 27, Apr.May.Jun. 25, Jul.Aug.Sep. 23, Oct.Nov.Dec. 17 